
\title{
28 Research Suare \\ Long Noncoding RNA LncPGCR mediated by TCF7L2 regulates chicken PGCs formation
}

\author{
Jingyi Jiang \\ Yangzhou University \\ Qisheng Zuo \\ Yangzhou University \\ Shaoze Cheng \\ WuXi AppTec \\ Xia Yuan \\ Yangzhou University \\ Jing Jin \\ Yangzhou University \\ Chen Chen \\ Yangzhou University \\ Chen Zhang \\ Yangzhou University \\ Jing Zhou \\ Yangzhou University \\ Yani Zhang \\ Yangzhou University \\ Guohong Chen \\ Yangzhou University \\ Bichun Li ( $\mathbf{7 8 6 2 8 2 3 7 3 @ q q . c o m ) ~}$ \\ Yangzhou University
}

\section{Research}

Keywords: LncPGCR, PGCs, TCF7L2, Btrc, ceRNA, chicken

Posted Date: May 20th, 2020

DOI: https://doi.org/10.21203/rs.3.rs-27736/v1

License: (c) (1) This work is licensed under a Creative Commons Attribution 4.0 International License. Read Full License 


\section{Abstract}

Background: Several of the thousands of long noncoding RNAs (IncRNAs) have been functionally characterized, yet its specific function and molecular mechanism in the formation of chicken primordial germ cells (PGCs) remains poorly understood. In the present study, we aim to investigate the role of LncPGCR (LncRNA PGCs Regulator) in PGCs formation.

Methods: LncPGCR, Cvh, Nanog, C-kit, gga-mir-6577-5p and Btrc expressions were detected by qRT-PCR. The percentage of PGCs cells was detected by flow cytometry, immunocytochemistry, and PAS staining. The interaction of histone acetylation, DNA methylation, transcription factor TCF7L2, and LncPGCR was confirmed by Luciferase reporter assay. The interaction of GAPDH and LncPGCR was measured by RNA pull-down, RIP, and Western blot.

Results: We observed the increased expression of LncPGCR in PGCs. It is mainly expressed in the cytoplasm and encodes small peptides. Moreover, over-expression of LncPGCR could promote PGCs formation in vitro and in vivo. Besides, we first reported that histone acetylation, DNA methylation, and transcription factor TCF7L2 can regulate LncPGCR expression in PGCs. In addition, LncPGCR activates WNT signaling pathways to promote PGCs formation by adsorbing gga-mir-6577-5p, relieving its inhibitory effect on target gene Btrc. Meanwhile, LncPGCR contributed to PGCs formation by increasing the phosphorylation level of GAPDH to activate the TGF- $\beta$ signal pathway.

Conclusion: LncPGCR over-expression promoted ESCs differentiation into PGCs through the potential LncPGCR/miR-6577-5p/BTRC pathway or increasing the phosphorylation level of GAPDH to activate the TGF- $\beta$ signal pathway.

\section{Background}

Primordial germ cells (PGCs) are primordial progenitor cells of germ cells and play a widespread role in the research of the preparation of gonadal chimeras, production of transgenic animals, and genomic imprinting. Moreover, PGC's multidirectional differentiation potential and unique regeneration ability make it one of the most promising seed cells in clinical medicine and tissue engineering research. However, the system for inducing PGCs in vitro is still immature. Due to its complex induction system and low induction efficiency, it is impossible to obtain sufficient cells for scientific research. Over the past decades, mounting evidences have emphasized that the induction of paracrine signaling(Saitou, Barton et al. 2002, Zhao and Garbers 2002), inhibition of somatic fate(Ohinata, Payer et al. 2005), alteration of epigenetic marks(Ancelin, Lange et al. 2006), and maintenance of pluripotency(Kehler, Tolkunova et al. 2004) are important for the formation of PGCs. However, the induction efficiency of PGCs in vitro has not been substantially improved, so we must conduct innovative exploration in the unknown field to obtain sufficient PGCs.

Current evidences demonstrate that LncRNA can participate in cell differentiation by interacting with microRNA(Cesana, Cacchiarelli et al. 2011), recruiting proteins as scaffolds to form complexes(Tsai, 
Manor et al. 2010), and trapping transcription factors(Guttman, Garber et al. 2010, Mohamed, Gaughwin et al. 2010). Nevertheless, the detail molecular mechanism of LncRNA during ESCs differentiation into PGCs is still sustain unclear. With the availability and rapid development of RNA-seq analysis technology, thousands of LncRNAs have been identified in the chicken genome in the past few years(Bao, Wu et al. 2013), and the function of LncRNA in embryonic development and germ cell differentiation has been preliminarily understood, but the specific regulatory mechanism has not been elucidated.

In this study, LncRNA expression patterns of chicken ESCs, PGCs, and SSCs were detected from singlecell levels by RNA-seq. PGCs-specific LncRNAs (TCONS_00948124) were identified and named LncPGCR (LncRNA PGCs Regulator). LncPGCR is activated by the transcription factor TCF7L2, histone acetylation, and DNA methylation during the formation of PGCs. Moreover, LncPGCR was also shown to regulate the gga-mir-6577-5p targeted gene BTRC by functioning as a competitive endogenous RNA (ceRNA) for ggamir-6577-5p, thereby promoting the expression of BTRC and PGCs formation. At the same time, LncPGCR can interact with GAPDH to activate the TGF- $\beta$ signal to regulate the formation of PGCs. Here we systematically studied the roles and molecular mechanisms of LncPGCR in PGCs formation.

\section{Materials And Methods}

\subsection{Antibody and reagent}

Commercially available antibodies were used: anti-C-Kit (Southern Biotech), anti-DDX4/CVH (ab13840, Abcam); GAPDH Anti-Phosphoserine (SPM101, Abcam); Goat Anti-Mouse IgG (Cy3 labeled, Bio-Synthesis, Inc., Texas, USA;dilution ratio:1:100).

Recombinant Human Bone Morphogenetic Protein-4 (BMP4, cyt-361) is from PROSPEC Dulbecco's modified Eagle medium (DMEM. 41965062) and Fetal bovine serum (FBS. 10100-147) from Gibco; FuGENE® HD (E2311) and Dual-Luciferase® Reporter Assay System from Promega; Leukemia Inhibit Factor from mouse (mLIF L5158), Fibroblast Growth Factor-Basic human (bFGF, F0291), Stem Cell Human (SCF. S7901); 5-aza-2'-deoxycytidine and TSA from Sigma; Pierce RNA 3' end desthiobiotinylation kit from Thermo; RIP-Assay Kit (RN1001) from MBL; PARIS ${ }^{\mathrm{TM}}$ from Ambion; PAS / Glycogen Stain kit (D004-1-2) from Nanjing Jiancheng Bioengineering Institute.

\subsection{Isolation and culture of ESCs, PGCs, and SSCs}

The study employed freshly fertilized eggs from Rugao yellow chickens, provided by the Poultry Research Institute of the Chinese Academy of Agricultural Sciences. ESCs were derived from 0-d-old chicken embryos, PGCs from the genital ridge of 4.5-d-old embryos, and SSCs were from testes of 18.5-d-old embryos. Hatching conditions were $37.5^{\circ} \mathrm{C}$ and $65 \%$ relative humidity. For specific separation and cultivation, refer to Zhang's article(Zhang, Wang et al. 2017).

\subsection{Isolation of nuclear and cytoplasmic RNA}


Operations were performed according to the manufacturer's protocols of PARIS ${ }^{\mathrm{TM}}$ system Protein and RNA Isolation System; add $500 \mu \mathrm{L}$ Cell Disruption Buffer to every $10^{7}$ PGCs cells, and incubate on ice for 10 mins; After centrifugation at $500 \times \mathrm{g}$ for 5 mins at $4^{\circ} \mathrm{C}$, the supernatant is cytoplasm and the precipitate is the nucleus. After the sample is dissolved, RNA extraction is performed.

\subsection{Preparation of LncPGCR constructs}

Three shRNA knockdown target sites were designed and combined to the linear vector pGMLV-SC5. shRNA target sites sequence is shown in Supplementary Table.4. The constructed knockdown vector was transfected with DF1 for activity verification, and the most active vector was encapsulated with lentivirus; A pair of primers was designed to amplify the full-length CDS region of LncPGCR. The fragment was cloned into pcDNA3.0 plasmid digested with $\mathrm{Kpnl}$ and EcoRI to construct an overexpression vector and verify its activity. PCR primers are shown in Supplementary Table.6.

\subsection{Chicken embryo vascular injection}

Under sterile conditions, use tweezers to open a round hole with a diameter of $1-1.5 \mathrm{~cm}$ in the blunt end of the early embryo eggs (13-17HH) incubated for 48-58h. Find the position of the embryo under a stereomicroscope. Then, using a micropipette, fill the lentiviral vector with a final polybrene concentration of $8 \mathrm{ng} / \mu \mathrm{L}$ or the pcDNA 3.0-PGCR vector wrapped with PEl into glass injection needles and inject them into the blood vessels of chicken embryos; then add $20 \mu \mathrm{L}$ of Streptomyces penicillin vaccinate to the injection site, and finally cross-sealed with medical tape to continue hatching; Chicken embryos were collected at $4.5 \mathrm{~d}$ and observe the embryos under a stereo fluorescence microscope.

\subsection{Quantitative Real-time PCR (qRT-PCR)}

Total RNA was extracted using Trizol (TIANGEN, Beijing, China) and reverse-transcribed into CDNA with the Quantscript RT Kit (TIANGEN, Beijing, China). Gene expression was determined using an ABI PRISM 7500 fluorescent quantitative PCR instrument (Applied Biosystems, Carlsbad, California). qRT-PCR primers are shown in Supplementary Table 5, the internal reference gene: $\beta$-actin, the number of repetitions: $n=3$.

\subsection{Immunocytochemistry (ICC)}

$6 \mathrm{~d}$ ESCs in each group were fixed with $4 \%$ paraformaldehyde for 30 minutes, and then treated with $0.5 \%$ TritonX-100 for 15 minutes. After blocking for 2 hours in the dark, and then added with Cvh antibody. After incubating at $37{ }^{\circ} \mathrm{C}$ for 2 hours, overnight at $4{ }^{\circ} \mathrm{C}$; Then adding anti-mouse IgG, incubate at $37{ }^{\circ} \mathrm{C}$ in the dark for 2 hours; Next, it was stained with $5 \mathrm{ng} / \mu \mathrm{L}$ DAPI for $15 \mathrm{~min}$, and finally, slides were plated with glycerin(50\% glycerol, 50\% PBS), and the image was sealed. FV1000 laser scanning confocal microscope (Olympus, Tokyo, Japan) was used to observed the samples.

\subsection{Flow cytometric}


For flow cytometry analysis, the cultures were mildly trypsinized and harvested from 24 well plates. The cells were washed and resuspended in PBS buffer, then add Cvh antibody for labeling, incubate at $4^{\circ} \mathrm{C}$ overnight or incubate at $37^{\circ} \mathrm{C}$ for $1-2 \mathrm{~h}$; Finally, data were analyzed on a Flow cytometer (Becton Dickinson, San Jose, CA) with the Flowjo program.

\subsection{PAS staining}

The paraffin sections after dewaxing and rehydration were stained according to the PAS / Gycogen Stain kit (D004-1-2) of Nanjing Jiancheng Technology Co., Ltd.

\subsection{Plasmids}

The LncPGCR promoter region and each deleted fragment were designed and combined to the linear vector pGL3.0-basic. Primers are shown in Supplementary Table 1.

\subsection{Dual-luciferase assay}

pGL3/1033 and pLncPGCR-N1 plasmid was transfected into DF-1. Then 10 $\mu \mathrm{mol} / \mathrm{L}$ 5-aza-2'-deoxycytidine (5-Azadc) or $1 \mu \mathrm{mol} / \mathrm{L}$ Trichostatin A (TSA) was added in the cells. After $24 \mathrm{~h}$, cells were collected and luciferin was added using Dual-Luciferase Reporter Assay (Promega, USA).

\subsection{RNA pull-down}

Use Ambion's in vitro transcription kit, Maxiscript T7, for in vitro transcription, and perform RNA pull-down experiments according to Thermo's kit instructions to adsorb proteins that interact with LnCPGCR; The target proteins were separated on PAGE by vertical electrophoresis and then subjected to silver staining experiments. Design the sense and antisense PCR primers based on the LncPGCR sequence(Supplementary Table 6).

\subsection{RNA immunoprecipitation (RIP) assays}

RIP assays were performed using a GAPDH Anti-Phosphoserine (SPM101, Abcam), and operations were performed according to the manufacturer's protocols of RiboCluster ProfilerTM (RN1001, MBL)RIP-Assay kit. Western Blot was used to detect the expression of the phosphorylation level of GAPDH on the extracted cellular protein samples after RNA enrichment.

\subsection{Data analysis}

Relative gene expression was calculated using the $2^{-\Delta \Delta C t}$ method after PCR. All experiments were performed in triplicate, and the data are expressed as mean \pm standard error. Significant differences between the groups were determined with two-sample t-tests in SPSS 18.0. $\left({ }^{*}, P<0.05\right.$, significant difference. ${ }^{\star \star}, P<0.01$, extremely significant difference).GraphPad Prism7 software was used for mapping.

\section{Results}




\subsection{LncPGCR, a PGCs specific LncRNA in Chickens, mainly expressed in the cytoplasm}

We analyzed RNA-seq data for IncRNAs from chicken ESCs, PGCs, and SSCs. Results showed that more than $75 \%$ of DELs were enriched in biological processes (Figure 1A). RT-PCR revealed that the expression of PGCs specifically expressed LncRNA was consistent with the sequencing results, indicating that the sequencing results were accurate and available(Supplementary Figure 1A). GO and KEGG enrichment analysis PGCs specifically expressed IncRNA(Supplementary Figure 1B,C), and excavated IncRNAs that are simultaneously enriched in the differentiation of ESCs into germ cells and germ cells differentiationrelated signaling pathways. We noticed that LncRNA (TCONS_00948124) is specifically expressed in PGCs (Table 1). Then, we assessed LncPGCR expression in chicken tissues. Results showed that LncRNA was significantly higher in chicken gonads than in other tissues (Supplementary Figure 1D). These data demonstrated high LncPGCR expression was closed related to the development of PGCs, so we named it LncPGCR (LncRNA PGCs Regulator).

LncRNA exhibits distinct molecular mechanisms and functions due to their different expression positions. We found that LnCPGCR was expressed in both the nucleus and the cytoplasm of the PGCs, but the expression level in the cytoplasm was significantly higher than that in the nucleus, which indicated that the mechanism of LncPGCR action mainly existed post-transcriptional level (Figure 1B). There are four ORFs in the body region of LncPGCR, of which ORF-2 encodes a small peptide, which may be involved in the formation of PGCs in this way (Figure 1C). These results indicate that LnCPGCR is a gene that is highly expressed in the cytoplasm of PGCs and encodes a protein.

\subsection{LncPGCR promotes ESCs differentiation into PGCs in vitro}

Then, we investigated the role of LncPGCR on PGCs growth. We transfected the LncPGCR overexpression/knockdown vectors based on the RA induction model and observed the morphology of ESCs cells in each group on the 2nd and 4th days. We noticed that in the normal induction model, the cells started to appear Embryoid Body (EB) on the 2nd day, and the EB began to increase and grow on the 4th day. After over-expression of LncPGCR, large EB appeared on the 2nd day, and small gaps began to appear on the edge of the embryoid body of type $4 \mathrm{~d}$. However, knockdown with LncPGCR delayed the appearance of EB and did not appear on the 4th day (Figure 2A). Meanwhile, the expression of Nanog decreased significantly after over-expression of LncPGCR $(P<0.05)$, while the expression of cvh and c-kit increased significantly $(P<0.01)$; Reciprocally, knockdown of LncPGCR decreased cvh and c-kit expression and increased the expression of Nanog(Figure 2B). Also, immunocytochemical and flow cytometry analysis confirmed that LncPGCR can promote the formation of PGCs (Figure 2C, D). These data demonstrated that LncPGCR can promote the formation and development of PGCs by inhibiting cell totipotence.

\subsection{LncPGCR promotes ESCs differentiation to PGCs in vivo}

To further verify the function of LncPGCR in the formation of PGC, we injected the PGCR-sh2 lentiviral vector and pcDNA3.0-PGCR vector into the embryonic blood vessels after 2.5 days of chicken embryo 
hatching and continued to hatch. Collecting chicken embryos from the knockdown group when it hatched to $4.5 \mathrm{~d}$ found that it could excite green fluorescence, indicating that the injected knockdown vector could effectively integrate into the chicken embryo genome (Figure 3A). The formation of PGCs in the genital ridge was significantly reduced after knockdown LncPGCR through paraffin section observation (Figure 3B). At the same time, qRT-PCR showed that after knocking down LncPGCR, the expression of Nanog in the genital ridge of chicken embryos at $4.5 \mathrm{~d}$ increased significantly $(P<0.01)$, while the expression of cvh and c-kit decreased significantly $(P<0.01)$ (Figure 3C); flow cytometry analysis confirmed that the formation of PGCs was suppressed after knockdown LncPGCR (1\% \pm 0.19$)$ (Figure 3D), which was consistent with the results of induction in vitro. In summary, LncPGCR has a positive regulatory effect on the formation of chicken PGCs.

\subsection{Epigenetic factors regulate differential expression of LncPGCR}

LncPGCR played a vital role in the formation of PGC. Therefore, we set out to determine the regulators of LncPGCR. Firstly, we cloned the promoter of LncPGCR and replaced the CMV promoter of pEGFP-N1 (Figure 4A), and found that it had promoter activity after transfection into DF-1 (Supplementary Figure $2 \mathrm{~A})$; Dual-luciferase assay revealed that the $-1033 \sim-661 \mathrm{bp}$ region was the core active region of the LncPGCR promoter(Figure 4B).

To further explore the transcriptional regulation mechanism of the LncPGCR, we first used The JASPAR database to predict the $-1033 \sim-661 \mathrm{bp}$ region and found that there is a binding site for the transcription factor TCF7L2 (Figure 4C). The dual-luciferase assay revealed that the TCF7L2 binding site mutation significantly reduced the LncPGCR promoter activity $(P<0.01)$, indicating that the transcription factor TCF7L2 is a positive regulator of the LncPGCR promoter (Figure 4D), and the expression of TCF7L2 was up-regulated during the induction of ESCs into PGCs in vitro (Figure 4E), suggesting that TCF7L2 is a key factor for the specific expression of LncPGCR in PGCs.

Epigenetics is essential for regulating gene transcription(Sewack, Ellis et al. 2001, Takeshima, Wakabayashi et al. 2014). Deacetylation and methylation of chromatin could silence the expression of genes. Dual-luciferase assay verified that 5-Azadc and TSA can significantly increase the LncPGCR promoter activity $(P<0.01)$ (Figure $4 \mathrm{~F})$. Collectively, these data demonstrate that the specific expression of LnCPGCR in PGCs is regulated by DNA methylation, histone acetylation, and the transcription factor TCF7L2.

\subsection{LncPGCR facilitates the formation of PGCs partially through sponging gga-mir-6577-5p, and then up- regulating Btrc}

Bioinformatics analysis of the sequence of LnCPGCR found its target gene Btrc. The interaction between miRNA and LncRNA is a classic regulation mode, which is widely involved in various life activities. We further identified 3 miRNAs with common binding targets between LncPGCR and Btrc (Table 2). Based on their binding site information and bioinformatics scores, we chose gga-mir-6577-5p for verification. qRTPCR showed that LncPGCR can promote the expression of Btrc, while gga-mir-6577-5p can inhibit its 
expression (Figure 5A, B), confirming that Btrc is a common target gene of LncPGCR and gga-mir-6577$5 p$.

We noticed that gga-mir-6577-5p also inhibited the expression of LncPGCR (Figure 5C), suggesting that there is a potential CeRNA regulation mode among LncPGCR, gga-mir-6577-5p, and Btrc. Strikingly, when overexpressed LncPGCR in systems gga-mir-6577-5p inhibit Btrc expression, we found that Btrc expression rose back (Figure 5D). These results indicated that LncPGCR can promote the formation of PGCs by competitively adsorbing gga-mir-6577-5p to release Btrc.

\subsection{The complex of LncPGCR-GAPDH activates TGF- $\beta$ signaling to promote the formation of PGCs}

Interactions between RNA and protein is one of the decisive factors for the realization of cellular physiological processes(Lukong, Chang et al. 2008, Castello, Fischer et al. 2013). To find proteins that interact with LncPGCR, we screened 23 protein interaction pairs through RNA pull-down combined with LC-MS / MS mass spectrometry, including GAPDH, KRT5, KRT19, LOC776816, etc. Among them, the interaction proteins GAPDH attracted our attention (Table 3, Figure 6A). Researches report that GAPDH can negatively regulate PKM2 to affect TGF- $\beta$ signaling pathway transduction(Hjerpe, Brage et al. 2013, Yanling 2014). Moreover, our previous studies have also confirmed that the TGF- $\beta$ signaling pathway can promote the formation of PGCs(Zuo, Jin et al.). Then the question arises: what role does LncPGCR play in the transduction of the TGF- $\beta$ signaling pathway? In this study, RIP and Western Blot were performed, and GAPDH protein phosphorylation was inhibited after knockdown LncPGCR (Figure 6B). These results indicate that $L$ CPGCR can induce the phosphorylation of GAPDH to activate the TGF- $\beta$ signal to regulate the formation of PGCs.

\section{Discussion}

In this study, we identified a key LnCRNA (LnCPGCR) in promoting ESCs differentiate into PGCs. Here, we showed that LncPGCR was highly expressed in gonads tissues, and higher LncPGCR expression was closed associated with PGCs formation. We also confirmed that LncPGCR can promote PGC formation in vivo and in vitro. Further, our data also imply that LnCPGCR' roles in promoting PGCs formation are through sponging gga-mir-6577-5p, and then activating Btrc. Besides, LncPGCR can regulate the phosphorylation level of GAPDH protein and activate TGF- $\beta$ signal to regulate the formation of PGCs.

Epigenetic modification can regulate differential expression of LncPGCR. Previous studies have shown that histone acetylation can change the structure of nucleosomes, keep the chromatin conformation open, and promote the binding of transcription factors to chromosomal DNA, which is conducive to gene transcription and expression. And DNA methylation can inhibit gene expression by changing chromatin structure( $\mathrm{Ng}$ and Bird 1999). Here, we find that reducing the level of DNA methylation or increasing the level of histone acetylation can promote the transcription of LnCPGCR, which is consistent with previous studies. What is interesting to us is that the transcription factor TCF7L2 can target the regulation of LncPGCR expression. Transcription Factor 7-like-2囚TCF7L2\, also known as T-cell transcription factor 4 (TCF-4), is a transcription factor containing a DNA-binding domain. Previous studies have shown that 
TCF7L2 is an important component of the Wnt signaling pathway(Kwak, Cho et al.). Moreover, our previous studies have verified that Wnt signaling pathways are involved in regulating the differentiation of ESCs to SSCs(He, Wang et al. 2017). It was also found that TCF7L2 can affect the formation of PGCs as a key transcription factor downstream of the Wnt signaling pathway(data not published), suggesting that LnCPGCR is regulated by WNT signals to participate in the formation of PGCs.

LncRNA can be used as a molecular sponge of endogenous miRNA, and its molecular regulatory mechanism through binding to miRNA is the ceRNA mechanism(Sun, Nie et al. 2016), this mechanism has received the attention of academic circles since it was proposed and it represents a new gene expression regulation model. Wang found that linc-ROR can regulate human ESC maintenance and differentiation, and subsequent sequence and function analysis found that because linc-ROR shared miRNA response elements with core transcription factors such as Oct4, Sox2, and Nanog, Linc-ROR acts

as a sponge during ESC cell differentiation, preventing these core transcription factors from being miRNAmediated inhibition(Wang, Xu et al.). Here, we found that LnCPGCR showed similar functions, which could adsorb gga-mir-6577-5p, improve Btrc expression during PGCs formation.

Mostly IncRNAs function by interacting with corresponding RNA-binding proteins, such as IncRNA TERRA through interaction with hnRNPA1 to achieve cell immortality(Oliva-Rico and Herrera 2017). In our study, we found that LncPGCR can effectively regulate the phosphorylation level of GAPDH protein.

Furthermore, it was found that GAPDH can negatively regulate PKM2 to affect the transduction of the TGF- $\beta$ signaling pathway, and the TGF- $\beta$ signaling pathway can promote the differentiation of ESCs to PGCs(Zuo, Jin et al.). These findings suggest that LnCPGCR can participate in the development of PGCs by regulating the TGF- $\beta$ signaling pathway. The above results indicate that LnCPGCR may exert various molecular mechanisms and play a key role in PGCs development.

\section{Conclusion}

In summary, LncPGCR acts as an adsorption sponge to release the inhibitory effect of gga-mir-6577-5p on BTRC. At the same time, the interaction between LncPGCR and its recruited protein GAPDH activates the TGF- $\beta$ signal and promotes PGCs production. The results of this study elaborated on the function and molecular mechanism of LncPGCR in the formation of PGCs and provided a theoretical basis for further improving the molecular network of LncRNA regulating PGCs formation.

\section{Abbreviations}

ESCs: embryonic stem cells; PGCs: primitive germ cells; SSCs: spermatogonial stem cells;5-Azadc: 5-aza2'-deoxycytidine; TSA: TrichostatinA; EB: Embryoid Body;

\section{Declarations}

\section{ACKNOWLEDGEMENTS}


We thank the Poultry Institute of the Chinese Academy of Agricultural Sciences Experimental Poultry Farm for providing experimental materials.

\section{FUNDING}

This study was supported by grants from the Key Research and Development

Program(2017YFE0108000), National Natural Science Foundation of China (31772582, 31872341 and 31572390), High Level Talents Support Program of Yangzhou University, Jiangsu Science and Technology Project (Youth Fund): BK20180918; Natural Science Research Project of Jiangsu Higher Education Institutions: 18KJB230008.

\section{ETHICS APPROVAL}

The procedures involving animals and their care conformed to the U.S. National Institute of Health guidelines (NIH Pub. No. 85-23. revised 1996). The experiments were conducted under the approval of the Ethics Committee of Yangzhou University for Laboratory and Experimental Animals (YZUDWSY2017-1215). The chickens used in this study were obtained from the Institute of Poultry Science, Chinese Academy of Agriculture Sciences. The experimental procedures were approved by the Institutional Animal Care and Use Committee of Yangzhou University.

\section{COMPETING INTERESTS}

The authors declare that they have no competing of interest

\section{CONSENT FOR PUBLICATION}

Written informed consent for publication was obtained from all participants.

\section{AVAILABILITY OF DATA AND MATERIALS}

All data generated or analyzed during this study are available from the corresponding author upon reasonable request.

\section{AUTHOR CONTRIBUTIONS}

B.C.L conceptualized the research program. B.C.L and G.H.C designed the experiments and coordinated the project. J.Y.J and S.Z.C performed the experiments. J.Y.J analyzed the data. J.Y.J and Q.S.Z wrote the manuscript. J.Y.J, Q.S.Z, S.Z.C, X.Y, J.J, C.C, C.Z, J.Z, Y.N.Z, G.H.C and B.C.L edited the manuscript. All authors discussed the results and commented on the manuscript.

\section{References}

1. Ancelin, K., U. C. Lange, P. Hajkova, R. Schneider, A. J. Bannister, T. Kouzarides and M. A. Surani (2006). "Blimp1 associates with Prmt5 and directs histone arginine methylation in mouse germ 
cells." Nature cell biology 8(6): 623-630.

2. Bao, J., J. Wu, A. S. Schuster, G. W. Hennig and W. Yan (2013). "Expression profiling reveals developmentally regulated IncRNA repertoire in the mouse male germline." Biology of reproduction 89(5): 107, 101-112.

3. Castello, A., B. Fischer, M. W. Hentze and T. Preiss (2013). "RNA-binding proteins in Mendelian disease." Trends in Genetics 29(5): 318-327.

4. Cesana, M., D. Cacchiarelli, I. Legnini, T. Santini, O. Sthandier, M. Chinappi, A. Tramontano and I. Bozzoni (2011). "A long noncoding RNA controls muscle differentiation by functioning as a competing endogenous RNA." Cell 147(2): 358-369.

5. Guttman, M., M. Garber, J. Z. Levin, J. Donaghey, J. Robinson, X. Adiconis, L. Fan, M. J. Koziol, A. Gnirke and C. Nusbaum (2010). "Ab initio reconstruction of cell type-specific transcriptomes in mouse reveals the conserved multi-exonic structure of lincRNAs." Nature biotechnology 28(5): 503.

6. He, N., Y. Wang, C. Zhang, M. Wang and B. Li (2017). "Wnt Signaling Pathway Regulates Differentiation of Chicken Embryonic Stem Cells into Spermatogonial Stem cells via Wnt5a." Journal of Cellular Biochemistry 119(2).

7. Hjerpe, E., S. E. Brage, J. Carlson, M. F. Stolt and E. Avall-Lundqvist (2013). "Metabolic markers GAPDH, PKM2, ATP5B and BEC-index in advanced serous ovarian cancer." Bmc Clinical Pathology 13(1): 30.

8. Kehler, J., E. Tolkunova, B. Koschorz, M. Pesce, L. Gentile, M. Boiani, H. Lomelí, A. Nagy, K. J. McLaughlin and H. R. Schöler (2004). "Oct4 is required for primordial germ cell survival." EMBO reports 5(11): 1078-1083.

9. Kwak, S. H., Y. M. Cho, M. K. Moon, J. H. Kim, B. L. Park, H. S. Cheong, H. D. Shin, H. C. Jang, S. Y. Kim and H. K. Lee "Association of polymorphisms in the insulin-degrading enzyme gene with type 2 diabetes in the Korean population." Diabetes Research \& Clinical Practice 79(2): 0-290.

10. Lukong, K. E., K.-W. Chang, E. W. Khandjian and S. Richard (2008). "RNA-binding proteins in human genetic disease." Trends in Genetics 24(8): 416-425.

11. Mohamed, J. S., P. M. Gaughwin, B. Lim, P. Robson and L. Lipovich (2010). "Conserved long noncoding RNAs transcriptionally regulated by Oct4 and Nanog modulate pluripotency in mouse embryonic stem cells." Rna 16(2): 324-337.

12. Ng, H. H. and A. Bird (1999). "DNA methylation and chromatin modification." Current Opinion in Genetics \& Devolopment 9(2): 158-163.

13. Ohinata, Y., B. Payer, D. O'Carroll, K. Ancelin, Y. Ono, M. Sano, S. C. Barton, T. Obukhanych, M. Nussenzweig and A. Tarakhovsky (2005). "Blimp1 is a critical determinant of the germ cell lineage in mice." Nature 436(7048): 207-213.

14. Oliva-Rico, D. and L. A. Herrera (2017). "Regulated expression of the IncRNA TERRA and its impact on telomere biology." Mechanisms of ageing and development 167: 16-23.

15. Saitou, M., S. C. Barton and M. A. Surani (2002). "A molecular programme for the specification of germ cell fate in mice." Nature 418(6895): 293-300. 
16. Sewack, G. F., T. W. Ellis and U. Hansen (2001). "Binding of TATA binding protein to a naturally positioned nucleosome is facilitated by histone acetylation." Molecular and cellular biology 21(4): 1404-1415.

17. Sun, M., F. Nie, Y. Wang, Z. Zhang, J. Hou, D. He, M. Xie, L. Xu, W. De and Z. Wang (2016). "LncRNA HOXA11-AS promotes proliferation and invasion of gastric cancer by scaffolding the chromatin modification factors PRC2, LSD1, and DNMT1." Cancer research 76(21): 6299-6310.

18. Takeshima, H., M. Wakabayashi, N. Hattori, S. Yamashita and T. Ushijima (2014). "Identification of coexistence of DNA methylation and H3K27me3 specifically in cancer cells as a promising target for epigenetic therapy." Carcinogenesis 36(2): 192-201.

19. Tsai, M.-C., O. Manor, Y. Wan, N. Mosammaparast, J. K. Wang, F. Lan, Y. Shi, E. Segal and H. Y. Chang (2010). "Long noncoding RNA as modular scaffold of histone modification complexes." Science 329(5992): 689-693.

20. Wang, Y., Z. Xu, J. Jiang, C. Xu, J. Kang, L. Xiao, M. Wu, J. Xiong, X. Guo and H. Liu "Endogenous miRNA Sponge lincRNA-RoR Regulates Oct4, Nanog, and Sox2 in Human Embryonic Stem Cell SelfRenewal." Developmental Cell 25(1): 69-80.

21. Yanling, C. (2014). PKM2 regulates cell motility through EGF/EGFR and TGFB/TGFßR signal pathways in hepatocellular carcinoma cells. Master, Fujian Medical University.

22. Zhang, C., M. Wang, N. He, M. F. Ahmed and B. Li (2017). "Hsd3b2 Associated in Modulating Steroid Hormone Synthesis Pathway Regulates the Differentiation of Chicken Embryonic Stem Cells into Spermatogonial Stem cells." Journal of Cellular Biochemistry 119(1).

23. Zhao, G.-Q. and D. L. Garbers (2002). "Male germ cell specification and differentiation." Developmental cell 2(5): 537-547.

24. Zuo, Q., K. Jin, Y. Zhang, J. Song and B. Li "Dynamic Expression and Regulatory Mechanism of TGF- $\beta$ Signaling in Chicken Embryonic Stem Cells Differentiating into Spermatogonial Stem Cells." Bioscience Reports: BSR20170179.

\section{Tables}

Table1 Differences in Specific Expression Related to Germ Cell Differentiation in PGCs IncRNA 


\begin{tabular}{llll} 
& InCRNA & $\begin{array}{l}\text { Target } \\
\text { gene }\end{array}$ & KEGG \\
\hline 1 & TCONS_00612668 & BCL9L & - \\
\hline 2 & TCONS_00765266 & Lef-1 & Wnt signaling pathway,Adherens junction,Melanogenesis \\
\hline 3 & TCONS_00874170 & BMP4 & TGF-beta signaling pathway,Hedgehog signaling pathway \\
\hline 4 & TCONS_00072199 & LIG4 & Non-homologous end-joining \\
\hline 5 & TCONS_00362261 & MAPK1 & TGF-beta signaling pathway,MAPK signaling pathway \\
\hline 6 & TCONS_00362344 & TBX3 & -- \\
\hline 7 & TCONS_00612668 & DDX6 & RNA degradation \\
\hline 8 & TCONS_00659989 & NR2E1 & - \\
\hline 9 & TCONS_00946310 & TRIM8 & TGF-beta signaling pathway \\
\hline 10 & TCONS_00580256 & SALL4 & - \\
\hline 11 & TCONS_00612668 & PHLDB1 & - \\
\hline 12 & TCONS_00947438 & TCF7L2 & Wnt signaling pathway,Adherens junction,Melanogenesis \\
\hline 13 & TCONS_00978804 & ACVR2A & TGF-beta signaling pathway \\
\hline 14 & TCONS_00948124 & BTRC & $\begin{array}{l}\text { TGF-beta signaling pathway,Hedgehog signaling } \\
\text { pathway,Oocyte meiosis }\end{array}$ \\
\hline 15 & TCONS_00627195 & IGF2BP1 & -- \\
\hline
\end{tabular}

Table 2 miRNA binding sites for LncPGCR and BTRC

\begin{tabular}{llll} 
miRNA name & Target gene & IncRNA ID & Target score \\
\hline gga-mir-6577-5p & BTRC & TCONS_0094124 & 93 \\
\hline gga-mir-6625-5p & BTRC & TCONS_0094124 & 91 \\
\hline gga-mir-3539 & BTRC & TCONS_0094124 & 85
\end{tabular}

Table 3 Mass Spectrometry Analysis of LncPGCR RNA Pull Down Protein 


\begin{tabular}{|c|c|c|c|}
\hline Numbers & Names & Coverage(\%) & Annotations \\
\hline 1 & KRT5 & 12.77 & Type II alpha-keratin IIA \\
\hline 2 & KRT6A & 16.19 & Uncharacterized protein \\
\hline 3 & RCJMB04_10b5 & 7.51 & Elongation factor 1 -alpha \\
\hline 4 & KRT19 & 15.36 & Keratin, type I cytoskeletal \\
\hline 5 & SERPING1 & 2.38 & Uncharacterized protein \\
\hline 6 & H4-VIII & 2.38 & Histone H4 type VIII \\
\hline 7 & HNRNPH3 & 3.71 & Uncharacterized protein \\
\hline 8 & HNRNPA2B1 & 4.41 & Uncharacterized protein \\
\hline 9 & LOC776816 & 8.75 & Uncharacterized protein \\
\hline 10 & LOC420039 & 6.86 & Uncharacterized protein \\
\hline 11 & & 1.68 & Histone H2A-III \\
\hline 12 & & 1.49 & Uncharacterized protein \\
\hline 13 & & 9.71 & Uncharacterized protein \\
\hline 14 & & 9.71 & Tubulin beta-7 chain \\
\hline 15 & ATP5B & 4.39 & ATP synthase subunit beta \\
\hline 16 & ACTG1 & 8.48 & Actin, cytoplasmic \\
\hline 17 & HSPA8 & 7.16 & Heat shock cognate $71 \mathrm{kDa}$ \\
\hline 18 & & 8.6 & $\begin{array}{l}\text { Heterogeneous nuclear ribonucleoprotein } \mathrm{H} 1 \text {-like } \\
\text { protein }\end{array}$ \\
\hline 19 & ILF3 & 7.21 & Interleukin enhancer binding factor 3-like protein \\
\hline 20 & & 6.98 & Uncharacterized protein \\
\hline 21 & LOC425049 & 7.03 & Tubulin alpha chain \\
\hline 22 & LOC420039 & 2.7 & Uncharacterized protein \\
\hline 23 & GAPDH & 17.41 & Glyceraldehyde-3-phosphate dehydrogenase \\
\hline
\end{tabular}

\section{Figures}




\section{A}

(a)

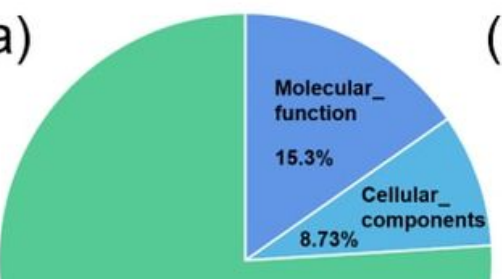

Biological_process $75.97 \%$

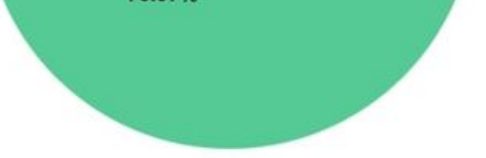

- Molecular hineson * cellular componerts = Biological prosess
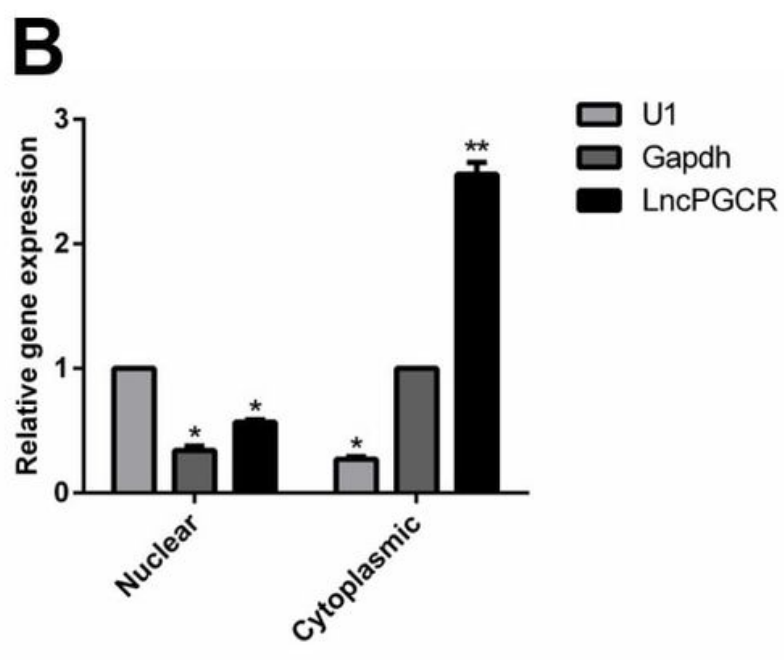

(b)

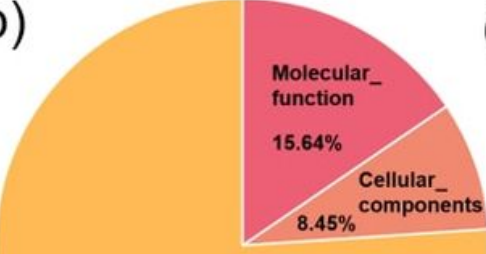

Biological_process $75.93 \%$

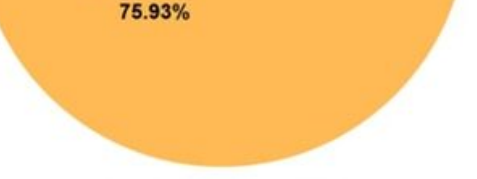

- Noliecular runction = collular components = blologieas proces: (c)
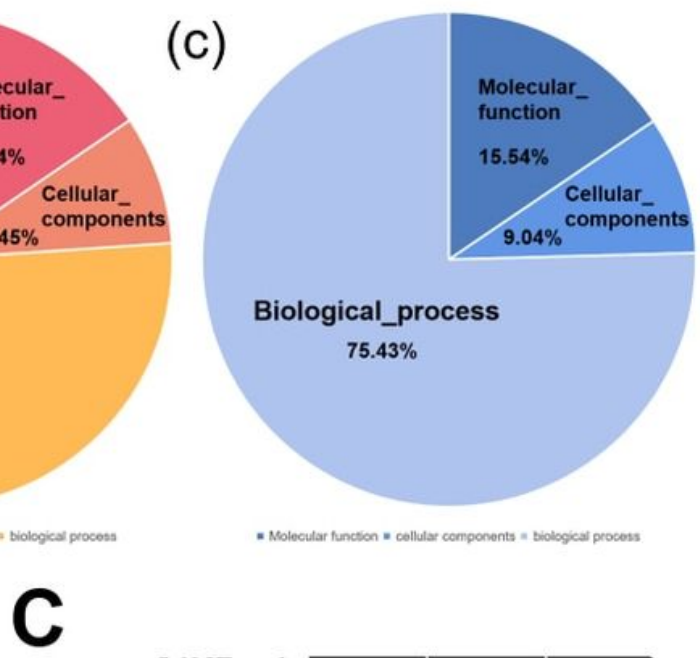

Biological_process $75.43 \%$

$75.43 \%$
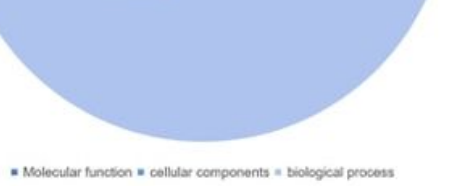

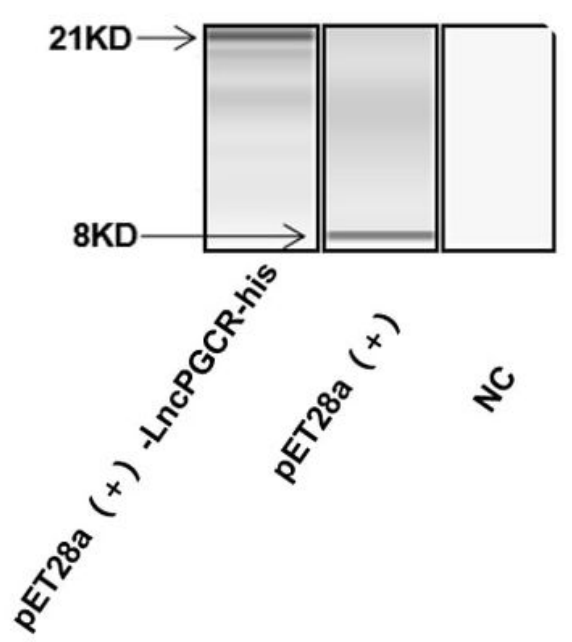

Figure 1

LncPGCR is a PGCs specific LncRNA in Chickens. A. GO enrichment analysis of LncRNAs differences functioned in ESCs vs PGCs(a), PGCs vs SSCs(b) and ESCs vs SSCs(c) as examined by RNA-seq. B. Levels of LncPGCR in the nucleus and cytoplasm of PGCs as measured by qRT-PCR. ${ }^{*} P<0.05$ compared with U1. The mean \pm s.e.m. is shown; $n=3$ independent experiments. C. Western Blot detects LncPGCR's ORF coding capabilities, LncPGCR 13kD + His 8kD. 
A

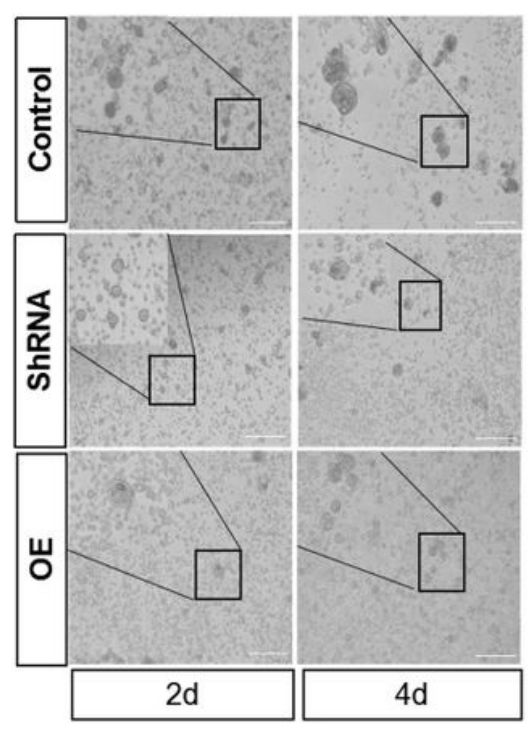

B
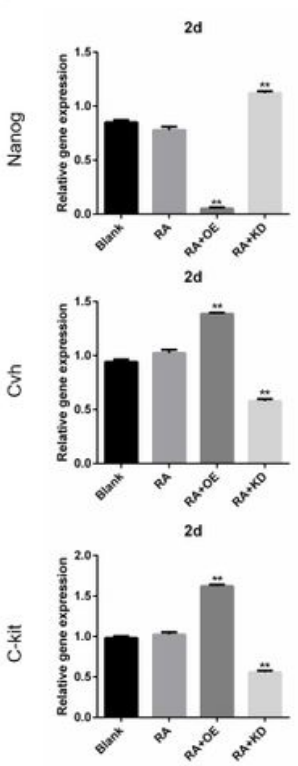
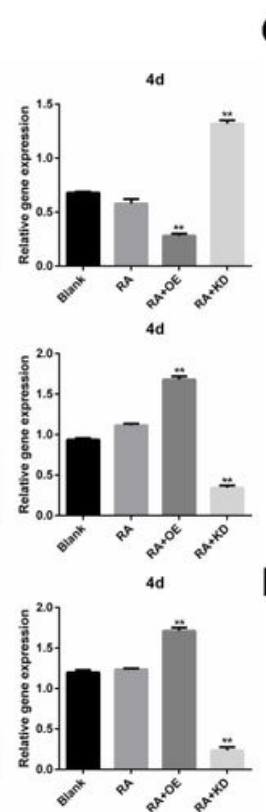

C
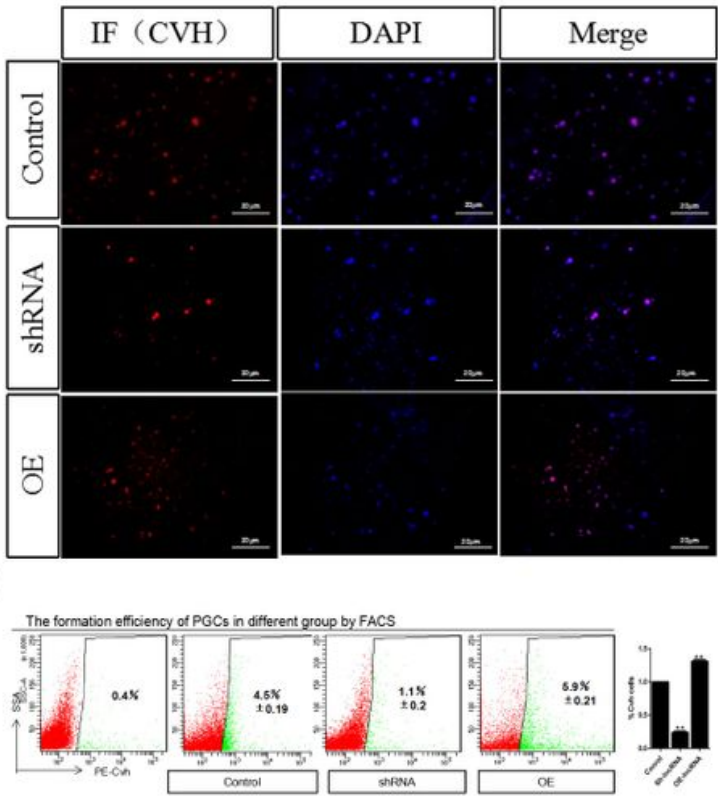

Figure 2

Ectopically expressed LncPGCR promotes ESCs differentiation into PGCs in vitro. A. Morphological changes of cells after overexpression or knockdown of LncPGCR in the RA induction model. B. qRT-PCR of Nanog, Cvh, and C-kit expression after overexpression or interference with LncPGCR in vitro. Values are means \pm SEM from three separate experiments. ${ }^{*} \mathrm{P}<0.05$ compared with untreated ESCs. $C$.

Immunocytochemical detection cvh expression on the 4th day after overexpression or interference with LncPGCR in the RA induction model in vitro. Scale bar: $20 \mu \mathrm{m}$. D. The proportion of d4 PGCs-like cells detected by flow cytometry analysis. 


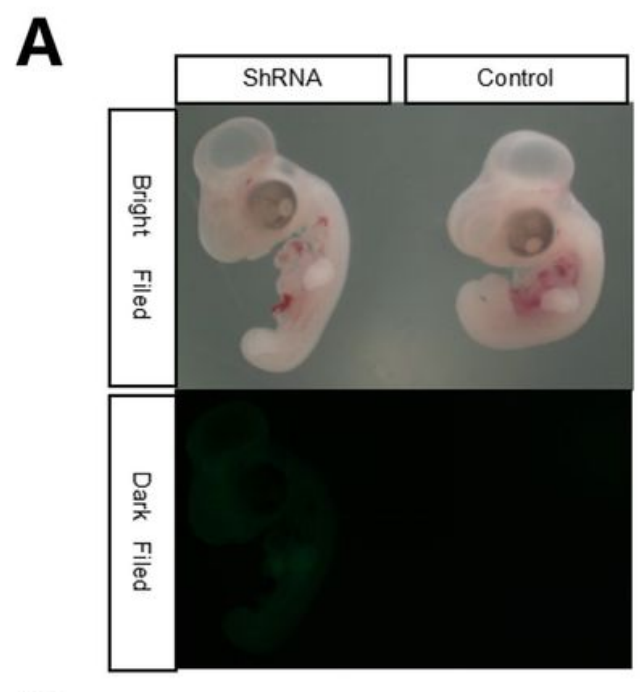

C

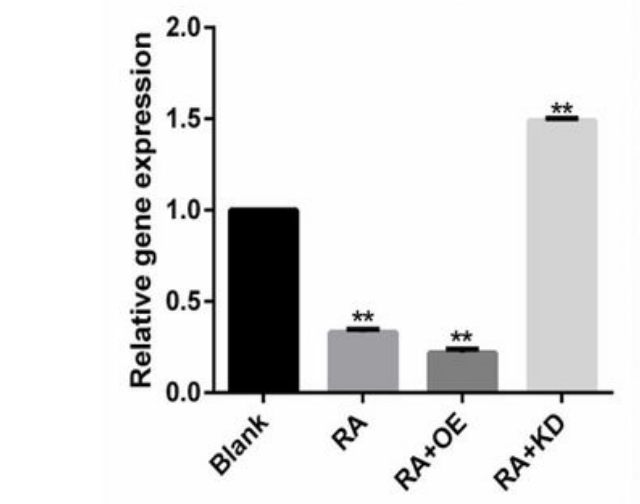

B

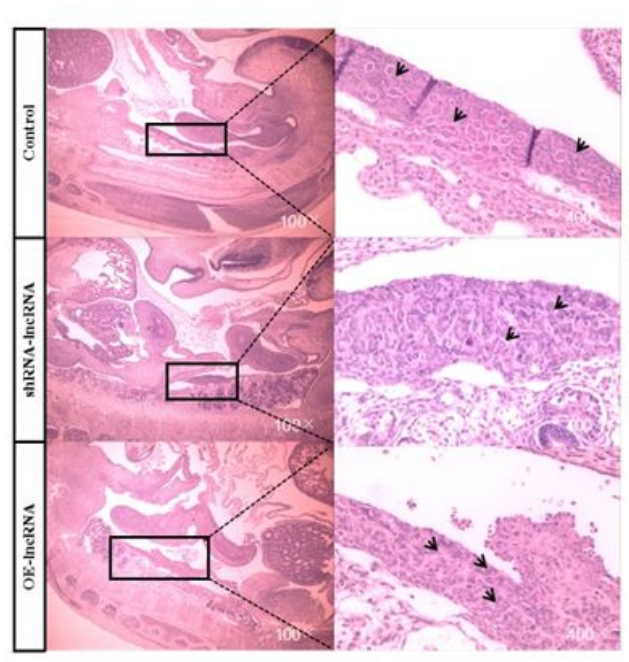

Cvh

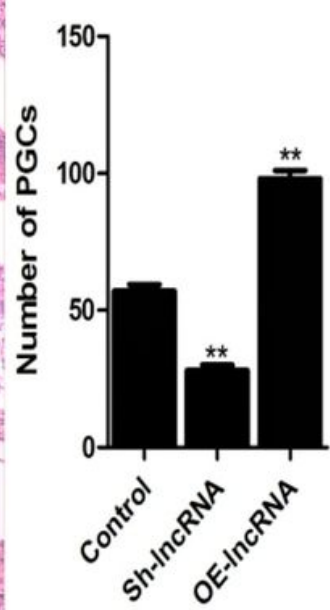

C-kit
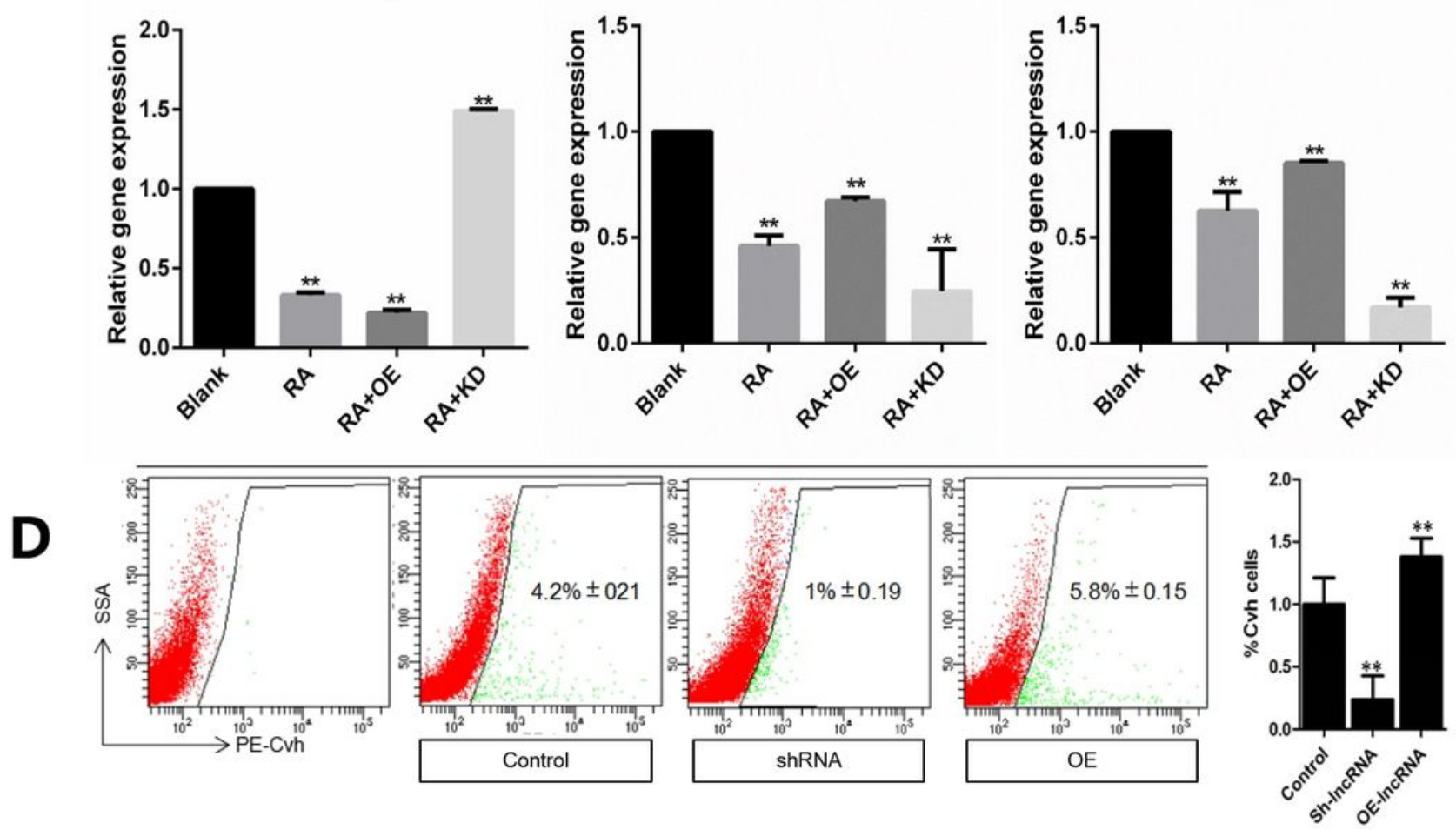

\section{Figure 3}

Ectopically expressed LncPGCR promotes PGCs formation in vivo. A.The result of EGFP expression in the chicken embryo. B. Photomicrographs of PAS staining of PGCs in the genital ridges. C. qRT-PCR of Nanog, Cvh, and C-kit expression after overexpression or interference with LncPGCR in vivo. Values are means \pm SEM from three separate experiments. ${ }^{*} P<0.05$ compared with the untreated chicken embryo. $D$. The proportion of PGCs-like cells detected by flow cytometry analysis. 
A

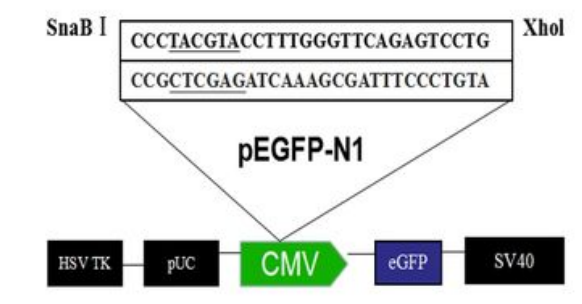

B

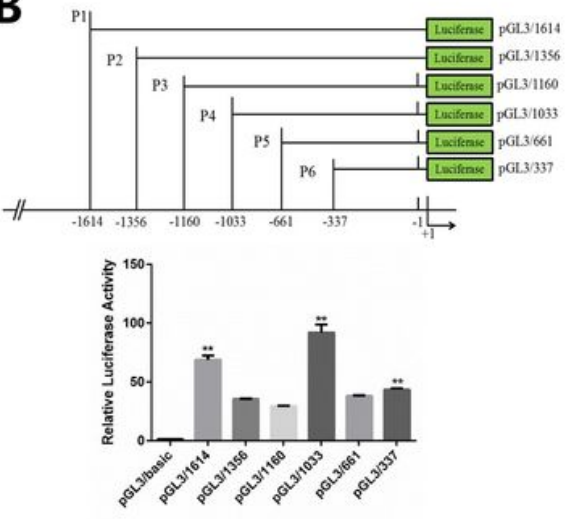

C

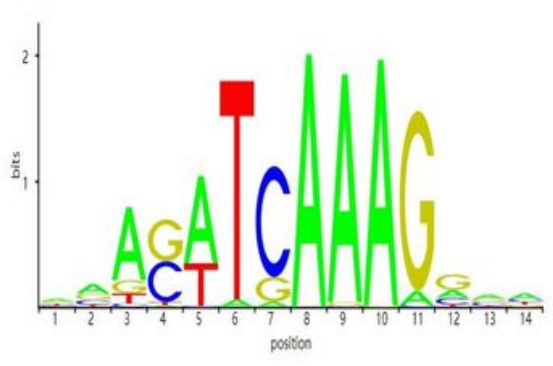

D

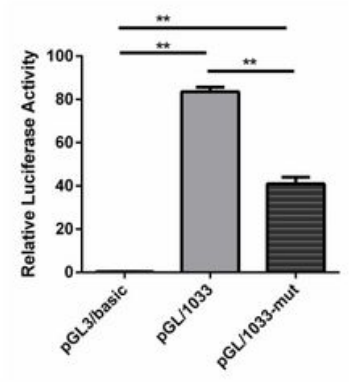

$\mathbf{E}$

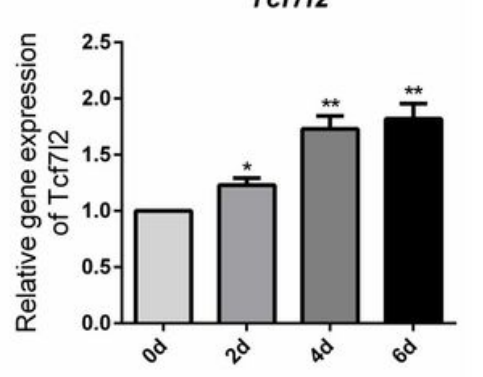

F (a)

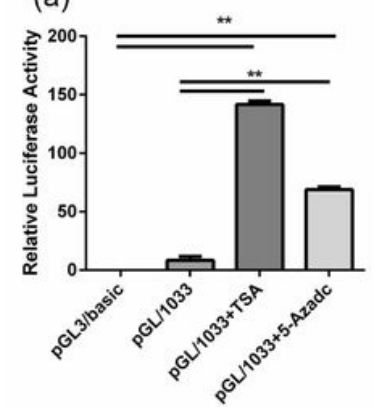

(b)

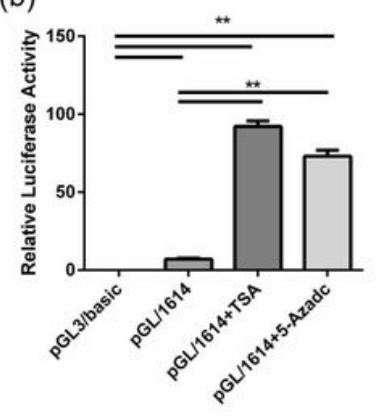

\section{Figure 4}

Histone acetylation, DNA methylation, and TCF7L2 regulate the expression of LncPGCR. A. Plasmid atlas of recombinant expression vector pLncPGCR-EGFP. B. Levels of LncPGCR promoter activity in DF1 cells, Values are means \pm SEM from three separate experiments. ${ }^{*} P<0.05$ compared with the control pGL3/BASIC vector. C. Schematic diagram of transcription factor TCF7L2 binding sites on LncPGCR. D. Levels of LncPGCR promoter activity in DF1 cells after mutation of the TCF7L2 binding site. Values are means $\pm S E M$ from three separate experiments. ${ }^{*} \mathrm{P}<0.05$ compared with the control pGL3/BASIC vector. $E$. qRT-PCR of Tcf7L2 expression during the induction of ESCs into PGCs in vitro.Values are means \pm SEM from three separate experiments. ${ }^{*} P<0.05$ compared with control ESCs. F. Levels of LncPGCR promoter activity in DF1 cells after adding 5-Azadc or TSA combined with the longest fragment pGL3/1033(a) and pGL3/1614(b). Values are means \pm SEM from three separate experiments. ${ }^{*} P<0.05$ compared with the control pGL3/BASIC vector. 
A

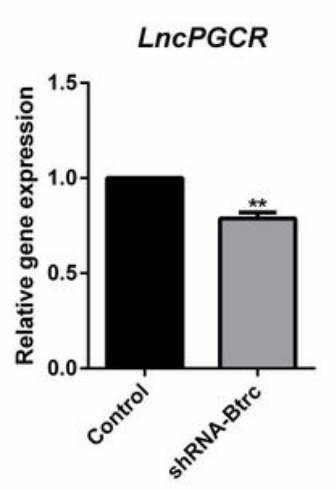

B

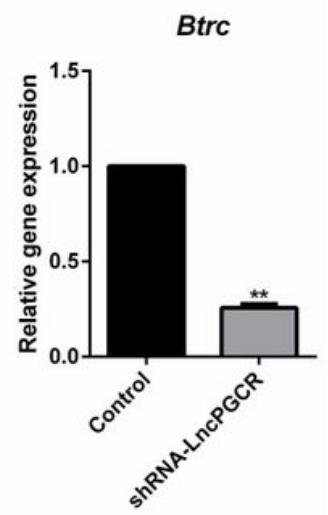

C

Btrc

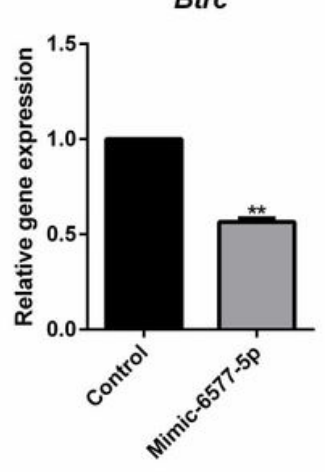

C D

LncPGCR

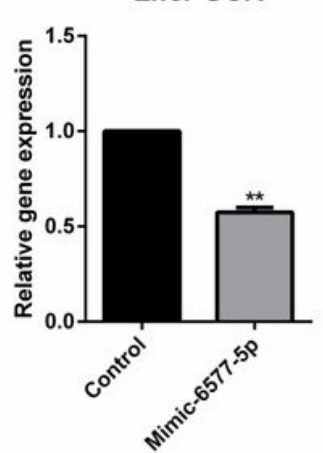

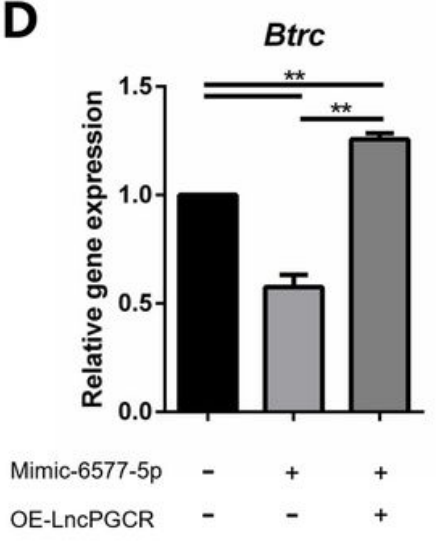

Figure 5

LncPGCR's activity is in part through negative regulation of gga-mir-6577-5p, and then activation of Btrc in PGCs. A. LncPGCR can promote Btrc expression as measured by qRT-PCR. Values are means \pm SEM from three separate experiments. ${ }^{*} P<0.05$ compared with control. B. gga-mir-6577-5p can inhibit Btrc expression as measured by qRT-PCR. Values are means \pm SEM from three separate experiments. ${ }^{*} P<0.05$ compared with control. C. gga-mir-6577-5p can inhibit LncPGCR expression as measured by qRT-PCR. Values are means \pm SEM from three separate experiments. ${ }^{*} P<0.05$ compared with control. $D$. Overexpression of LncPGCR can rescue Btrc expression after interfering with gga-mir-6577-5pas measured by qRT-PCR. Values are means \pm SEM from three separate experiments. ${ }^{*} P<0.05$ compared with control 
A
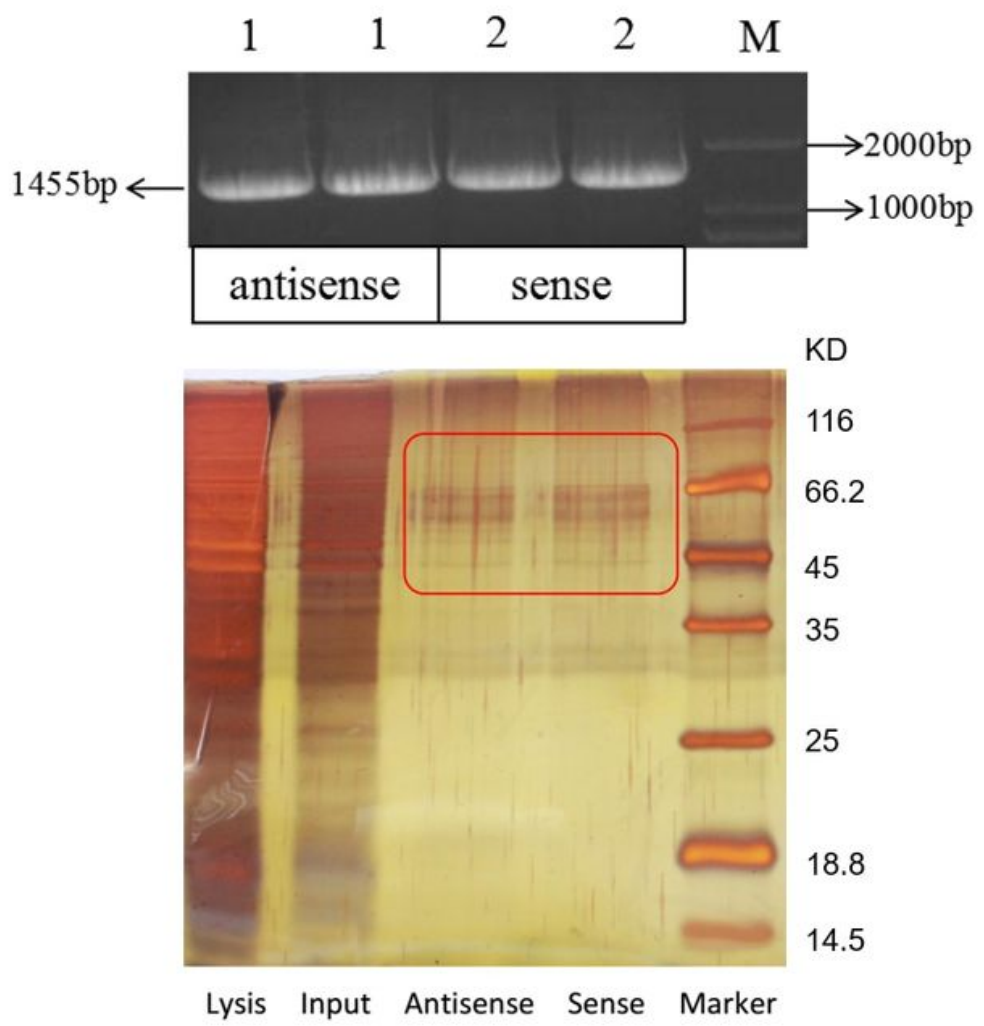

B

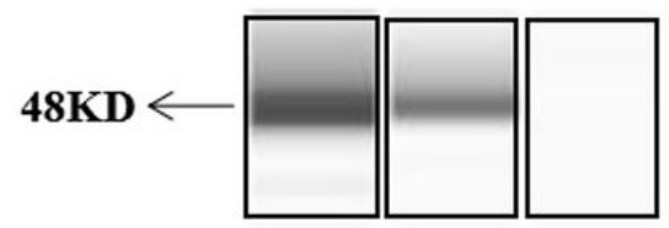

KD

116

66.2

45

35

25

18.8

14.5

\section{Figure 6}

LncPGCR can induce the phosphorylation of GAPDH to activate the TGF- $\beta$ signal. A. LncPGCR can interact with GAPDH by RNA pull-down. B. The knockdown of LncPGCR inhibits GAPDH protein phosphorylation by RIP.

\section{Supplementary Files}

This is a list of supplementary files associated with this preprint. Click to download.

- Tables.docx

- S.2.tif

- S.1.tif 